\title{
Shock-Driven Fluid-Structure Interaction for Civil Design
}

\author{
Stephen Wood \\ Research Alliance in Math and Science \\ Oak Ridge National Laboratory, \\ P.O. Box 2008 MS6367, \\ Oak Ridge, TN 37831, U.S.A.
}

\section{Acknowledgments}

This work was performed at the Oak Ridge National Laboratory, which is managed by UT-Battelle, LLC under Contract No. De-AC05-00OR22725. This work has been authored by a contractor of the U.S. Government, accordingly, the U.S. Government retains a nonexclusive, royalty-free license to publish or reproduce the published form of this contribution, or allow others to do so, for U.S. Government purposes. The author would like to thank Ms. Debbie McCoy for bringing him to ORNL through the Research Alliance in Math and Science (RAMS) program and the opportunity to conduct research with guidance from Dr. Ralf Deiterding. The author would also like to express his gratitude for the mentoring Dr. Deiterding has provided without which this work would not have been possible. Additionally, the aurthor would like to thank Dr. Emilian Popov for his input on the reactor building model.

\begin{abstract}
The multiphysics fluid-structure interaction simulation of shock-loaded structures requires the dynamic coupling of a shock-capturing flow solver to a solid mechanics solver for large deformations. The Virtual Test Facility combines a Cartesian embedded boundary approach with dynamic mesh adaptation in a generic software framework of flow solvers using hydrodynamic finite volume upwind schemes that are coupled to various explicit finite element solid dynamics solvers (Deiterding et al., 2006). This paper gives a brief overview of the computational approach and presents first simulations that utilize the general purpose solid dynamics code DYNA3D for complex 3D structures of interest in civil engineering. Results from simulations of a reinforced column, highway bridge, multistory building, and nuclear reactor building are presented.
\end{abstract}

Keywords: Fluid-structure interaction, Blast wave, Civil engineering 


\section{Table of Contents}

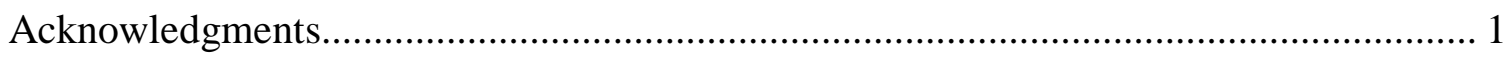

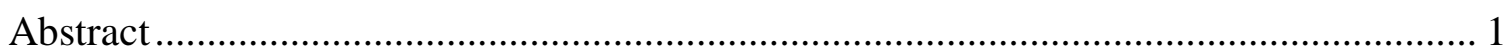

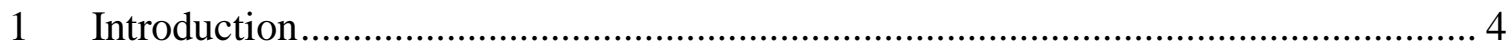

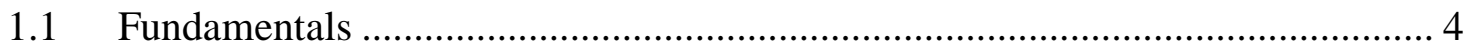

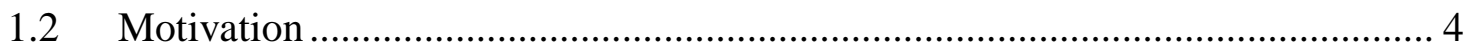

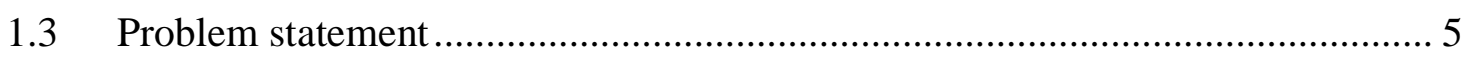

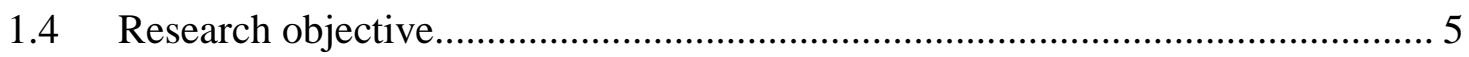

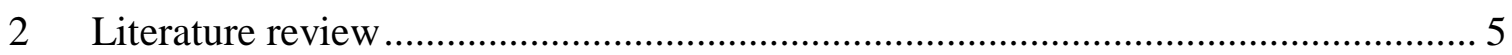

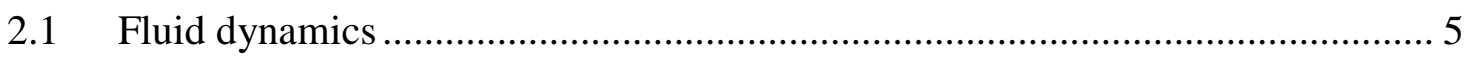

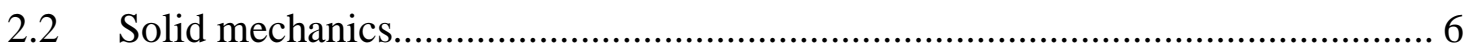

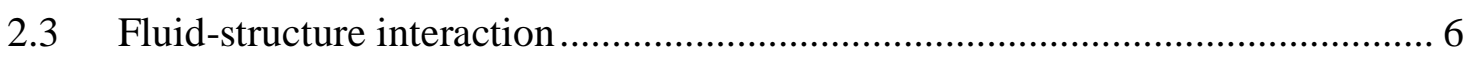

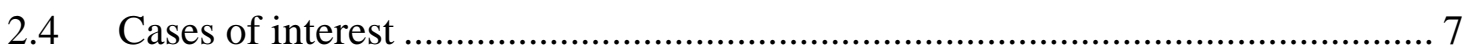

3 Physical problem and mathematical formulation .................................................... 7

3.1 Fluid governing equations and used upwind discretizations............................... 7

3.2 Embedding of moving boundaries into Cartesian schemes .................................. 8

3.3 Solid governing equations and material model..................................................... 9

3.4 Parallel block-structured adaptive mesh refinement (SAMR) ............................ 12

3.5 Multiphysics fluid-structure coupling with SAMR.............................................. 13

$4 \quad$ Results and discussion .................................................................................... 14

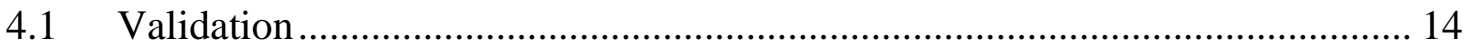

4.1.1 Single reinforced concrete column ………………................................... 14

4.1.2 Highway bridge................................................................................... 16

4.1.3 Multistory building ............................................................................. 19

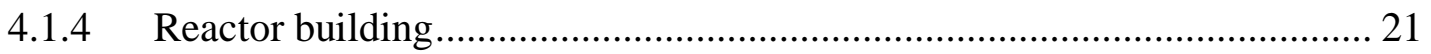

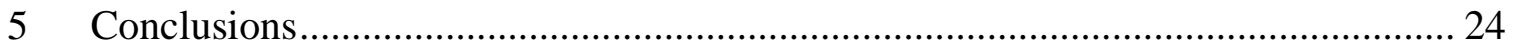

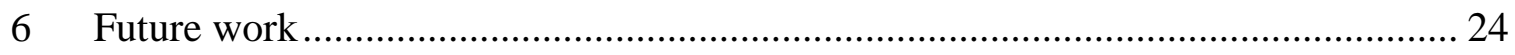

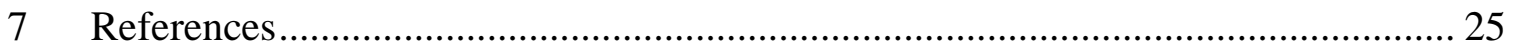




\section{Table of Figures}

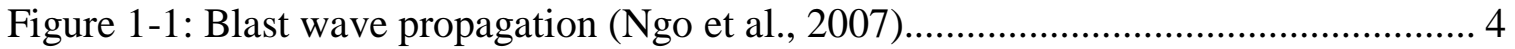

Figure 2-1: Blast loads on a building (Ngo et al., 2007).............................................. 5

Figure 2-2: Blast wave pressure time history (Ngo et al., 2007).................................... 5

Figure 3-2: Mapping of to fluid cell centers (dots) to solid surface cell centers (x).......... 8

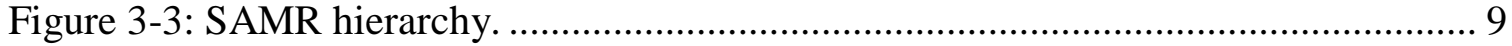

Figure 3-1: Uni-axial stress strain curve showing elastic-plastic material behavior for kinematic hardening $(\beta=0.0)$ and for isotropic hardening $(\beta=1.0)$ (Hallquist and Lin,

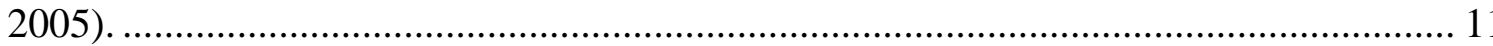

Figure 3-5: Flow of applied boundary pressure, location and velocity data exchanged between AMROC and DYNA3D at each time step through the Euler-Lagrangian Coupler (ELC).

Figure 4-1: Cross-section of column detailing rebar types and locations (400 mm ligature

spacing). 15

Figure 4-2:Air pressure shown on slice planes around deforming column (tan) shown in cutaway to reveal steel rebar (grey) for three time steps. ........................................... 15 Figure 4-3: Solid mesh convergence and comparison with simulation results by Ngo et al. (2007).

Figure 4-4: Plan and elevation of typical bridge (Agrawal and Yi, 2009).

Figure 4-5: Air pressure shown on slice plane above rigid ground (green) and around deforming bridge (tan) shown for initial time step.

Figure 4-6: Air pressure shown on slices of fluid domain and on the surfaces of the bridge and ground (bridge deck removed from view).

Figure 4-7: Detail of local failure in bridge deck and columns adjacent to detonation at $1.9 \mathrm{~ms}$.

Figure 4-8: Contour of air density gradient (red) shown around deforming building of high strength (yellow) and low strength (orange) concrete for the initial time step......... 20 Figure 4-9: Contour of air density gradient (red) shown around deforming building colored with surface pressure for two snapshots. 20

Figure 4-10: Detail of local failure of floors and walls adjacent to detonation at $8 \mathrm{~ms} . . .21$ Figure 4-11: Air pressure shown on slice plane around cutout of deforming building of high strength (orange) and low strength (yellow) concrete, girders (dark grey) and paneling (light grey) at initial time.

Figure 4-12: Air pressure shown on slice plane around cutout of deforming building of high strength (orange) and low strength (yellow) concrete, girders (dark grey) and paneling (light grey) $1.8 \mathrm{~ms}$ after detonation.

Figure 4-13: Air pressure shown on slice plane around cutout of deforming building of high strength (orange) and low strength (yellow) concrete, girders (dark grey) and paneling (light grey) $2.5 \mathrm{~ms}$ after detonation. 


\section{Introduction}

\subsection{Fundamentals}

The detonation of a condensed high explosive generates hot gases under pressure up to 300 kilobar and a temperature of about 3000-4000C ${ }^{\circ}$. The hot gas expands forcing out the volume it occupies. As a consequence, a layer of compressed air (blast wave) forms in front of this gas volume containing most of the energy released by the explosion. The blast wave instantaneously increases to a value of pressure above the ambient atmospheric pressure. This is referred to as the side-on overpressure that decays as the shock wave expands outward from the explosion source. After a short time, the pressure behind the front may drop below the ambient pressure (Figure 1-1). During such a negative phase air is sucked in from the surrounding area (Ngo et al., 2007).

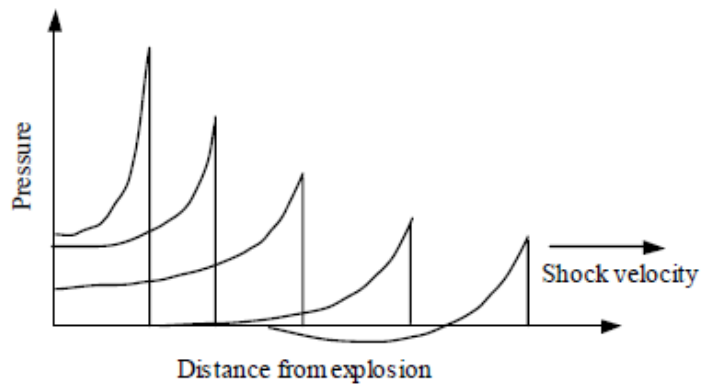

Figure 1-1: Blast wave propagation (Ngo et al., 2007).

The structural behavior of an object or structure exposed to such a blast wave may be analyzed by dealing with two main issues. Firstly, blast-loading effects, i.e., forces that result directly from the action of the blast pressure; secondly, the structural response, or the expected damage criteria associated with such loading effects. It is important to consider the interaction of the blast waves with the target structures. This might be quite complicated in the case of complex structural configurations. The structural response will depend upon the size, shape and weight of the target, how firmly it is attached to the ground, and also on the existence of openings in each face of the structure (Ngo et al., 2007).

\subsection{Motivation}

The use of vehicle bombs to attack city centers has been a feature of campaigns by terrorist organizations around the world. A bomb explosion within or immediately nearby a building can cause catastrophic damage on the building's external and internal structural frames, collapsing of walls, blowing out of large expanses of windows, and shutting down of critical life-safety systems. Loss of life and injuries to occupants can result from many causes, including direct blast-effects, structural collapse, debris impact, fire, and smoke. The indirect effects can combine to inhibit or prevent timely evacuation, thereby contributing to additional casualties. In addition, major catastrophes resulting from gaschemical explosions result in large dynamic loads, greater than the original design loads, of many structures. Due to the threat from such extreme loading conditions, efforts have been made during the past three decades to develop methods of structural analysis and design to resist blast loads. The analysis and design of structures subjected to blast loads require a detailed understanding of blast phenomena and the dynamic response of various structural elements. 


\subsection{Problem statement}

The design of buildings and structures to blast events is of critical importance in this era of improvised explosive devices. There is a lack of software capable of simulating these types of fluid-structure interactions.

Carrying out experiments to evaluate a structure's ability to withstand explosions is time consuming, costly and the results may only apply to a limited set of situations. Simulation software can provide a flexible means to examine and understand the performance of structures undergoing blast-induced fluid-structure interaction.

\subsection{Research objective}

The objective of this research is to validate the solvers AMROC (Adaptive Mesh Refinement in Object-oriented C++) and DYNA3D within the Virtual Test Facility fluidstructure interaction software suite with realistic structures subjected to blast conditions. Once validated, designers and analysts will have a valuable new tool for the investigation of blast-driven fluid-structure interaction.

\section{Literature review}

\subsection{Fluid dynamics}

The threat by a conventional bomb is defined by two equally important elements, the bomb size, or charge weight $W$, and the stand-off distance $R$ between the blast source and the target (Figure 2-1). For example, the blast that occurred at the basement of World Trade Centre in 1993 had the charge weight of $816.5 \mathrm{~kg}$ TNT. The Oklahoma bomb in 1995 had a charge weight of $1814 \mathrm{~kg}$ at a stand-off of $4.5 \mathrm{~m}$ (Longinow, 1996). As terrorist attacks may range from the small letter bomb to the gigantic truck bomb, as experienced in Oklahoma City, the mechanics of a conventional explosion and their effects on a target must be addressed. The observed characteristics of air blast waves are found to be affected by the physical properties of the explosion source.

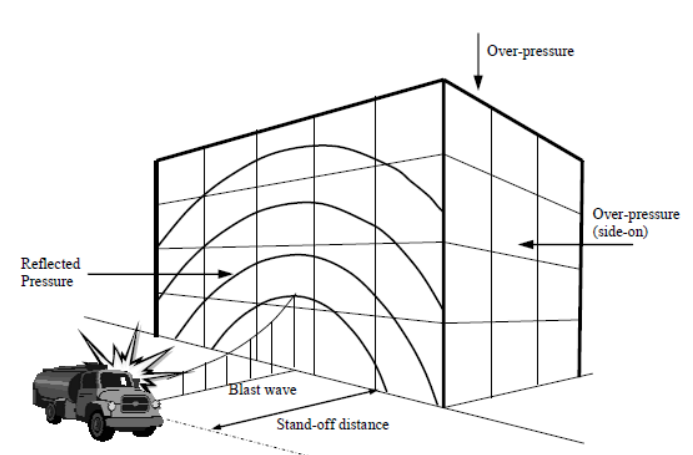

Figure 2-1: Blast loads on a building (Ngo et al., 2007).

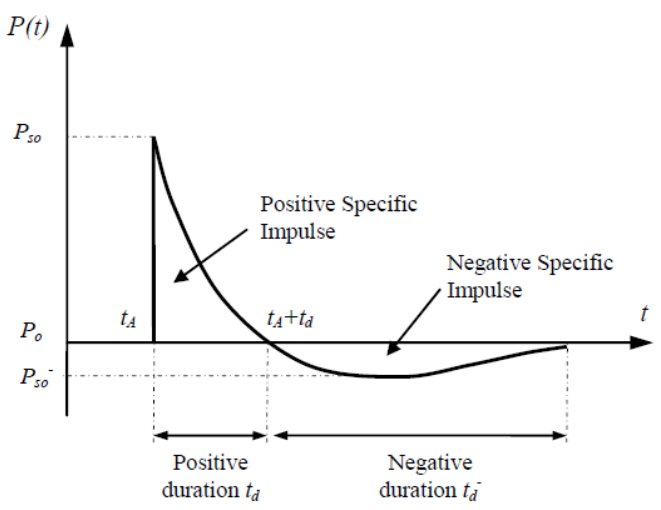

Figure 2-2: Blast wave pressure time history (Ngo et al., 2007).

Figure 2-2 shows a typical blast pressure profile. At the arrival time $t_{A}$, following the explosion, pressure at that position suddenly increases to a peak value of overpressure, $P_{s o}$, over the ambient pressure, $P_{o}$. The pressure then decays to ambient level at time $t_{d}$, then decays further to an under-pressure $P_{s o}$ - before eventually returning to ambient 
conditions at time $t_{d}+t_{d^{-}}$. The quantity $P_{\text {so }}$ is usually referred to as the peak side-on overpressure, incident peak overpressure or merely peak overpressure (TM 5-1300, 1990). The incident peak overpressure $P_{s o}$ is amplified by a reflection factor if the shock wave encounters an object or structure in its path. Except for specific focusing of high intensity shock waves at near $45^{\circ}$ incidence, these reflection factors are typically largest for normal incidence (a surface adjacent and perpendicular to the source) and decrease with the angle of obliquity or angular position relative to the source. Reflection factors depend on the intensity of the shock wave, and for large explosives at normal incidence these reflection factors may enhance the incident pressures by as much as a factor of two. Throughout the pressure-time profile, two main phases can be observed: The portion above ambient is called positive phase of duration $t_{d}$, while that below ambient is called negative phase of duration, $t_{d^{-}}$. The negative phase is of longer duration and lower intensity than the positive pressure phase. As the stand-off distance increases, the duration of the positive-phase blast wave increases resulting in a lower-amplitude, longer-duration shock pulse. Charges situated extremely close to a target structure impose a highly impulsive, high intensity pressure load over a localized region of the structure; charges situated further away produce a lower-intensity, longer-duration uniform pressure distribution over the entire structure. Eventually, the entire structure is engulfed in the shock wave, with reflection and diffraction effects creating focusing and shadow zones in a complex pattern around the structure. During the negative phase, the weakened structure may be subjected to impact by debris that may cause additional damage (Ngo et al., 2007).

\subsection{Solid mechanics}

Analyzing the dynamic response of blast-loaded structures involves considering the effect of high strain rates, the non-linear inelastic material behavior, the uncertainties of blast load calculations and the time-dependent deformations. Structural elements are expected to undergo large inelastic deformation under blast load or high velocity impact. Exact analysis of dynamic response is then only possible by numerical solution requiring nonlinear dynamic finite element software (Ngo et al., 2007).

DYNA3D is an explicit finite element code for analyzing the transient dynamic response of three-dimensional solids and structures. Many material models are available to represent a wide range of material behavior, including elasticity, plasticity, composites, thermal effects, and rate dependence. In addition, DYNA3D has a sophisticated contact interface capability, including frictional sliding and single surface contact, to handle arbitrary mechanical interactions between independent bodies (Hallquist and Lin, 2005).

\subsection{Fluid-structure interaction}

The Virtual Test Facility (VTF) is a software environment for coupling solvers for compressible computational fluid dynamics (CFD) with solvers for computational solid dynamics (CSD). The CFD solvers facilitate the computation of flows with strong shocks as well as fluid mixing. The CSD solvers provide capabilities for simulation of dynamic response in solids such as large plastic deformations, fracture and fragmentation. In addition, the VTF can be used to simulate highly coupled fluid-structure interaction problems, such as the high rate deformation of metallic solid targets forced by the loading from the detonation of energetic materials, or the rupture and fragmentation of brittle 
materials under shock wave impact. At present, all VTF solvers use time-explicit shockcapturing schemes (Deiterding et al., 2006).

\subsection{Cases of interest}

A structure impacted by blast often experiences loading from the initial blast front and from shocks reflected by its own surfaces, those of surrounding structures, and the ground. All of these loadings are capable of causing elastic and plastic deformation. This cumulative deformation can result in substantial damage and the collapse of the structure.

The reinforced concrete column is a common load bearing member in civil structures. A column's ability to withstand a blast event and continue to support the higher floors and roof of a building is crucial to maintaining the structural integrity of the building. As such the first case considered is that of a single reinforced concrete column impacted by a spherical blast. The second case investigated is that of a highway bridge subjected to a TNT detonation near one of its columns at a standoff distance of $0.5 \mathrm{~m}$. The third case considered is the progressive collapse of a multi-story building. A fourth case is considered to test the ability of AMROC-DYNA to accurately model a highly complex structure such as a reactor building.

\section{Physical problem and mathematical formulation}

\subsection{Fluid governing equations and used upwind discretizations}

The simulation of trans- or supersonic wave phenomena in fluids requires the consideration of the compressibility while viscosity can typically be neglected. The basic system of governing equations are the Euler equations:

$$
\begin{gathered}
\partial_{t} \rho+\nabla \cdot(\rho \boldsymbol{u})=0 \\
\partial_{t}(\rho \boldsymbol{u})+\nabla \cdot(\rho u \otimes \boldsymbol{u})+\nabla \mathrm{p}=0 \\
\partial_{t}(\rho E)+\nabla((\rho E+p) \mathbf{u})=0
\end{gathered}
$$

Herein, $\rho$ is the density, $\boldsymbol{u}$ the velocity vector and $E$ the specific total energy. In order to close (1), an equation of state $p=p(\rho, e)$ is required for modeling the dependency of the hydrostatic pressure $p$ on density $\rho$ and specific internal energy $e:=E-\frac{1}{2} \boldsymbol{u}^{T} \boldsymbol{u}$. For a single polytropic gas, the equation of state (EOS) reads

$$
p=(\gamma-1) \rho
$$

with $\gamma$ denoting the constant adiabatic exponent. For Eq. (2), the speed of sound in the fluid is $c=(\gamma p / \rho)^{1 / 2}$. The elastic-plastic deformation of structures hit by a shock wave in air, which is modeled as a polytropic gas with $\gamma=1.4$, is considered.

To solve Eqs. (1) and (2) numerically, we apply a time-explicit shock-capturing finite volume scheme based on Roe's approximate Riemann solver (Toro, 1999) specially hybridized with the Harten-Lax-vanLeer (HLL) scheme to ensure strict positivity 
preservation (see (Deiterding, 2003) for details). Second-order accuracy in smooth solution regions is achieved with the MUSCL-Hancock variable extrapolation technique.

\subsection{Embedding of moving boundaries into Cartesian schemes}

Geometrically complex moving boundaries are incorporated into the finite volume methods by using some of the cells as ghost cells for enforcing immersed moving wall boundary conditions, cf. (Arienti et al., 2003), (Fedkiw et al., 1999). The boundary geometry is mapped onto the Cartesian mesh by employing a signed level set function $\phi$ that stores the distance to the boundary surface and allows the efficient evaluation of the boundary outer normal in every mesh point as $\boldsymbol{n}=-\nabla \phi /|\nabla \phi|$ (Deiterding et al., 2006).

This coupling approach which utilizes disjointed computational domains allows ad-hoc separation in dedicated fluid and solid processors. Figure 3-2 shows the associations of fluid cell centers (dots) and solid cell centers (x) across the solid surface. The associations direct the mapping of solid surface nodes to fluid cells as indicated by the blue arrows.

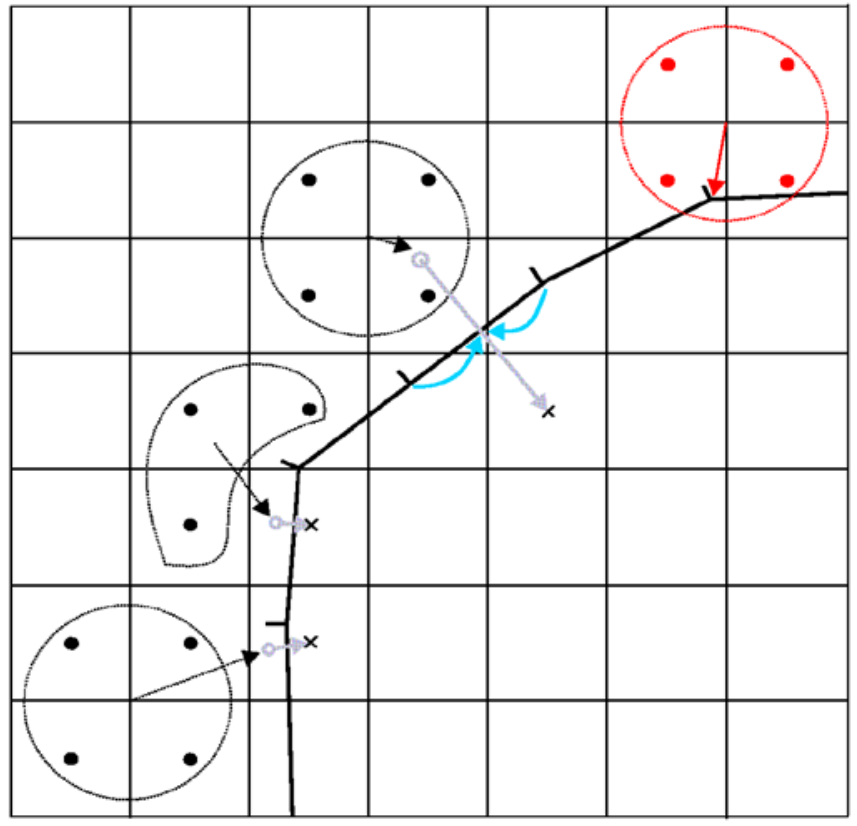

Figure 3-1: Mapping of to fluid cell centers (dots) to solid surface cell centers (x). 


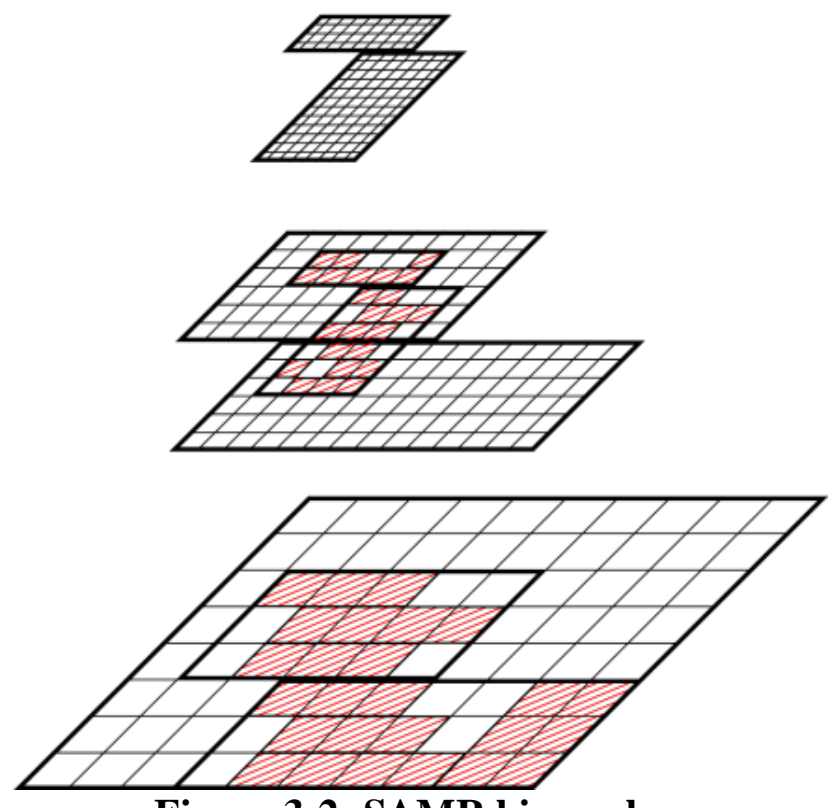

Figure 3-2: SAMR hierarchy.

For the governing equations (1), the boundary condition at a rigid wall moving with velocity $\boldsymbol{v}$ is $\boldsymbol{u} \cdot \boldsymbol{n}=\boldsymbol{v} \cdot \boldsymbol{n}$. Enforcing the latter with ghost cells, in which the discrete values are located at the cell centers, requires the mirroring of the values $\rho, \boldsymbol{u}, \boldsymbol{p}$ across the embedded boundary. The normal velocity in the ghost cells is set to $(2 \boldsymbol{v} \cdot \boldsymbol{n}-\boldsymbol{u} \cdot \boldsymbol{n}) \boldsymbol{n}$, while the mirrored tangential velocity remains unmodified. Mirrored values are constructed by calculating spatially interpolated values in the point $\tilde{\boldsymbol{x}}=\boldsymbol{x}+2 \phi \boldsymbol{n}$ from neighboring interior cells. We employ a dimension-wise linear interpolation for this operation, but it has to be emphasized that directly near the boundary the number of interpolants needs to be decreased to ensure the monotonicity of the numerical solution (Deiterding, 2009). This property is essential in simulating hyperbolic problems with discontinuities.

Crucial for the performance of the overall method is the fast evaluation of the distance information, which is computationally equivalent to determining for every fluid cell the closest solid mesh element. For this purpose, we employ a specially developed algorithm based on characteristic reconstruction and scan conversion (Mauch, 2003) that computes the accurate distance information only in a small band around the embedded structure.

\subsection{Solid governing equations and material model}

DYNA3D is based on a finite element discretization of the three spatial dimensions and a finite difference discretization of time. It uses a lumped mass formulation for efficiency. This produces a diagonal mass matrix $M$, which renders the solution of the momentum equation

$$
M \boldsymbol{a}_{n+1}=\boldsymbol{f}^{e x t}-\boldsymbol{f}^{\text {int }}
$$

trivial at each step in that no simultaneous system of equations must be solved. 
The basic continuum finite element in DYNA3D is the eight-node "brick" solid element. This element is valid for large displacements and large strains. The element may be degenerated to a wedge or tetrahedral element, but at the expense of accuracy. Thus, these degenerated elements should be avoided whenever possible (Lin 2005). The brick element type was found to be the most accurate solid element type for the high deformation and strain rates present in all test cases. DYNA3D also supports five quadrilateral 4-node shell elements which can be degenerated to a triangular three-node element, but at the expense of accuracy. The triangular elements formed from collapsed quadrilaterals were found to lock due to excessive transverse shear, yielding non-physical results. The quadrilateral four-node shell and eight-node "brick" elements produced results which are in good agreement with verification calculations based on the EulerBernoulli beam equation. The same element types produced results in good agreement with the experimental results for the plate deformation from water hammer experimental results. A full discussion of FSI verification and validation cases can be found in (Deiterding et al. 2006), (Deiterding, et al. 2009) and (Deiterding, 2010).

DYNA3D supports numerous material models suitable for a variety of materials and loading regimes. The kinematic/isotropic plasticity material model was well suited for the steel and copper structures of the verification and validation test cases because the shocks were severe enough to cause elastic and plastic deformation but not fracture.

The parameters of the kinematic/isotropic elastic-plastic model are the

$$
\begin{aligned}
& \text { Young's modulus, } E \\
& \text { Poisson's ratio, } v \\
& \text { Yield stress, } \sigma_{0} \\
& \text { Tangent modulus, } E_{T} \\
& \text { Hardening parameter, } \beta
\end{aligned}
$$

The yield condition of the model can be written as

$$
\phi=\bar{\sigma}-\sigma_{y}\left(\bar{\varepsilon}^{p}\right)
$$

where $\bar{\sigma}$ is the effective stress and $\sigma_{y}$ is the current yield stress, which may be a function of the effective plastic strain if strain hardening is included. For isotropic hardening, the effective stress $\bar{\sigma}$ is given by

$$
\bar{\sigma}=\frac{3}{2} s_{i j} s_{i j}{ }^{1 / 2}
$$

where $s_{i j}$ is the deviatoric stress tensor. For kinematic hardening,

$$
\bar{\sigma}=\frac{3}{2} \eta_{i j} \eta_{i j}{ }^{1 / 2}
$$

where the translated stress $\eta_{i j}$ is defined as 


$$
\eta_{i j}=s_{i j}-\alpha_{i j}
$$

and $\alpha_{i j}$ is the (deviatoric) back stress tensor.

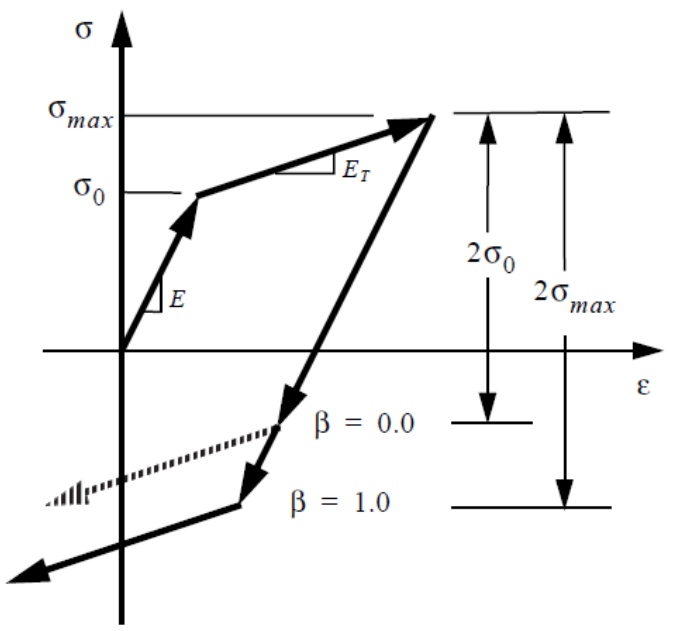

Figure 3-3: Uni-axial stress strain curve showing elastic-plastic material behavior for kinematic hardening $(\beta=0.0)$ and for isotropic hardening $(\beta=1.0)$ (Hallquist and Lin, 2005).

The linear isotropic hardening law has the form

$$
\sigma_{y}=\sigma_{0}+\beta E_{p} \bar{\varepsilon}^{p}
$$

where $\sigma_{y}$ is the current yield stress, $\sigma_{0}$ is the initial yield stress, and $E_{p}$ is the plastic modulus.

The uni-axial stress strain curve in Figure 3-1 shows the elastic-plastic material behavior for kinematic hardening $(\beta=0.0)$ and for isotropic hardening $(\beta=1.0)$.

The effective plastic strain is given by

$$
\bar{\varepsilon}^{p}=\int_{0}^{t} d \bar{\varepsilon}^{p}
$$

where the incremental effective plastic strain $d \bar{\varepsilon}^{p}$ is found from the incremental plastic strain tensor $d \bar{\varepsilon}_{i j}$ as

$$
d \bar{\varepsilon}^{p}=\frac{2}{3} d \bar{\varepsilon}_{i j}^{p} d \bar{\varepsilon}_{i j}^{p}
$$

The plastic modulus is found from Young's modulus $E$ and the tangent modulus $E_{T}$ using

$$
E_{p}=\frac{E E_{T}}{E-E_{T}}
$$


The plastic hardening modulus $E_{p}$ is the slope of the inelastic portion of the effective stress $\bar{\sigma}$ vs. effective plastic strain $\bar{\varepsilon}^{p}$ curve. Similarly, the tangent modulus $E_{T}$ is the slope of the inelastic part of a uniaxial stress $\bar{\sigma}$ vs. strain $\bar{\varepsilon}^{p}$ curve (or equivalently, the effective stress vs. effective strain curve).

Kinematic and isotropic hardening elastoplastic models yield identical behavior under monotonic loading. Under reversed loading from a maximum stress $\sigma_{\max }$, kinematic hardening predicts reverse yielding when the stress has unloaded by an amount $2 \sigma_{0}$, and isotropic hardening predicts that reverse yielding occurs when the stress reaches $-\sigma_{\max }$. Thus, under cyclic loading conditions where many stress reversals may occur, kinematic hardening predicts a hysteretic energy dissipation, while isotropic hardening predicts no energy dissipation after the first cycle. The isotropic model is slightly faster in computation speed, however (Lin, 2005).

The properties used to model standard concrete were:

$$
\begin{aligned}
& \text { Density, } \rho=2.010 e+03 \mathrm{~kg} / \mathrm{m}^{3} \\
& \text { Young's modulus, } E=2.172 e+10 \mathrm{~Pa} \\
& \text { Poisson's ratio, } v=2.000 e-01 \\
& \text { Yield stress, } \sigma_{0}=9.100 e+05 \mathrm{~Pa} \\
& \text { Tangent modulus, } E_{T}=1.120 e+10 \mathrm{~Pa} \\
& \text { Hardening parameter, } \beta=3.000 e-02
\end{aligned}
$$

\subsection{Parallel block-structured adaptive mesh refinement (SAMR)}

As it is characteristic for immersed Cartesian techniques, the boundary treatment described in the previous section results in some geometric approximation inaccuracies. We mitigate this problem by refining the embedded boundary dynamically during the computation, in most cases up to the highest available resolution. A refinement criterion based on $\phi \equiv 0$ has been implemented for this purpose.

In order to dynamically adapt the local resolution we employ the block-structured adaptive mesh refinement (SAMR) method after Berger and Colella (1988) which is tailored especially for hyperbolic conservation laws on logically rectangular grids. Cells being flagged by various refinement indicators, e.g., scaled gradients and/or heuristic error estimation by Richardson extrapolation, are clustered with a special algorithm into non-overlapping rectangular grids. The regularity of the grid data enables high computational performance on vector and super-scalar processors and cache optimizations. Values of cells covered by finer subgrids are overwritten by averaged fine grid values subsequently. Refinement grids are derived recursively from coarser ones and a hierarchy of successively embedded levels is thereby constructed, see Figure 3-3. Note also that the levels are integrated recursively using hierarchical time step refinement, which also contributes further to the efficiency of the approach. Spatial and temporal mesh widths on level $l$ are $r_{l}$-times finer than on level $l-1$, i.e. $\Delta t_{l}:=\Delta t_{l-1} / r_{l}$ and $\Delta x_{k, l}:=\Delta x_{k, l-1} / r_{l}$ with $r_{l} \geq 2$ for $l>0$ and with $r_{0}=1$, and a time-explicit finite volume scheme will (in principle) remain stable on all levels of the hierarchy. 
Parallelization of the SAMR method is relatively straightforward as already in the serial algorithm subgrids are computationally decoupled by utilizing layers of halo cells. The halos on level $l$ are set either to implement physical boundary conditions, for $l>0$ by time-space interpolation from data on level $l-1$, or by copying the data value from an overlying subgrid on $l$ (synchronization).In our SAMR solver framework AMROC we follow a rigorous domain decomposition approach and partition the SAMR hierarchy from the root level on. A careful analysis of the SAMR algorithm uncovers that the only parallel operations under this paradigm are halo cell synchronization, redistribution of the data hierarchy and the application of flux correction terms along internal refinement boundaries that impose the sum of abutting fine cell numerical fluxes on coarse grid cells (Deiterding, 2005). In AMROC, partitions with similar workload are found at runtime as the hierarchy evolves by a hierarchical partitioning algorithm based on a generalization of Hilbert's space-filling curve (Prashar \& Browne, 1996). The space-filling curve defines an ordered sequence on the cells of the root level that can easily be split in load-balanced portions. As such curves are constructed recursively, they are locality-preserving and therefore avoid an excessive data redistribution overhead. Further on, the surface area is small, which reduces synchronization costs. Recent AMROC benchmarks (not shown here) exhibit good scalability for typical SAMR fluid-only cases on several thousand processors.

\subsection{Multiphysics fluid-structure coupling with SAMR}

The fluid solver, AMROC (Adaptive Mesh Refinement in Object-oriented $\mathrm{C}++$ ), and the solid solver, DYNA3D, exchange data only at the interface between disjointed computational domains after consecutive time steps. Figure 3-5 shows the data flow within AMROC-DYNA from the initialization of the solution process to the iterative scheme of the coupled solver. The initialization parameters are listed within the hexagons at the top of the figure. 


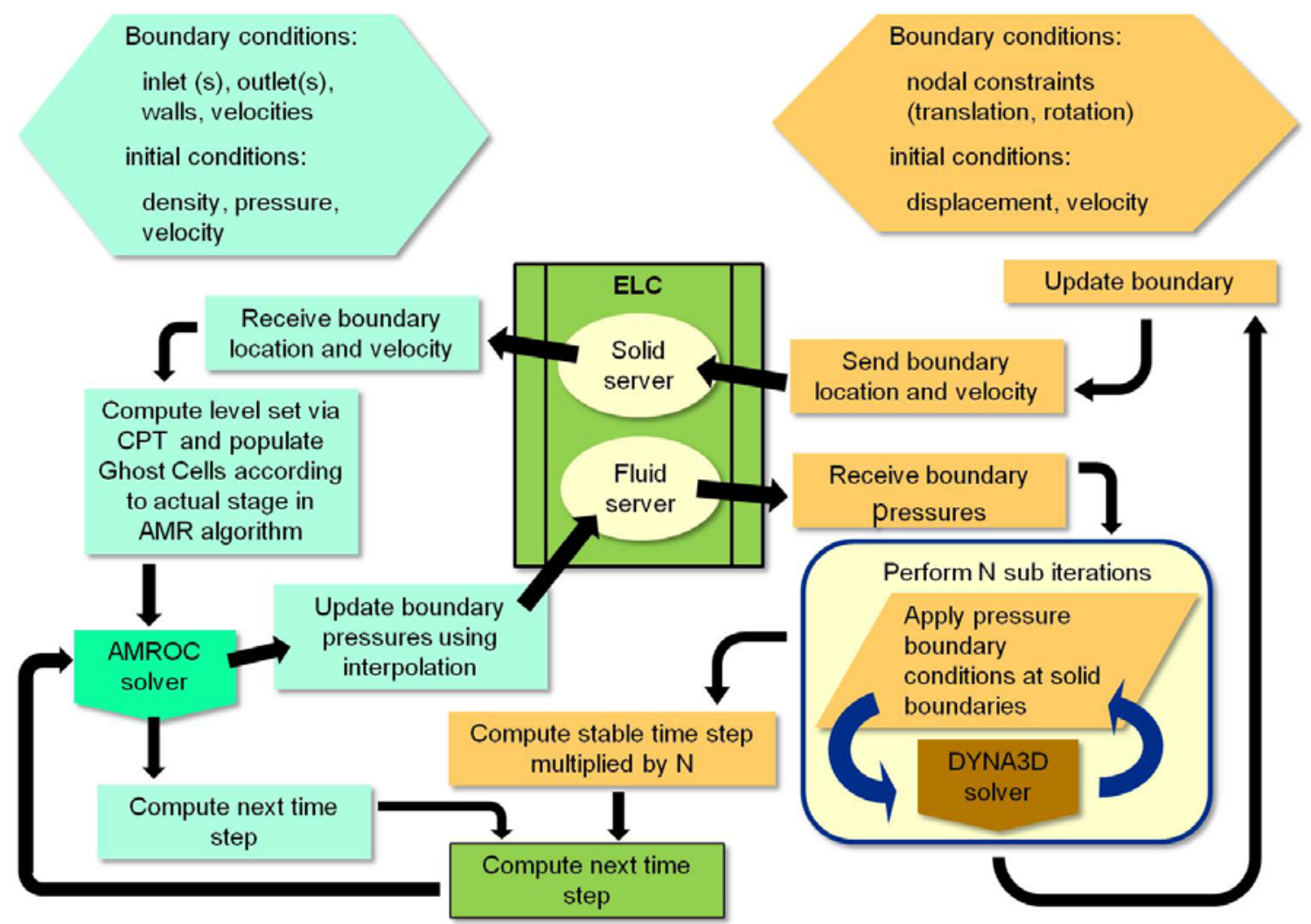

Figure 3-4: Flow of applied boundary pressure, location and velocity data exchanged between AMROC and DYNA3D at each time step through the Euler-Lagrangian Coupler (ELC).

\section{$4 \quad$ Results and discussion}

\subsection{Validation}

\subsubsection{Single reinforced concrete column}

This case study follows the setup described by Ngo et al. (2007) in Section 10 of their paper. The reinforced concrete column studied is $6.4 \mathrm{~m}$ tall with a $500 \mathrm{x} 900 \mathrm{~mm}$ crosssection and poured with 80MPa strength concrete. Figure 4-1 details the rebar types and locations in the column cross-section.

The blast loading is generated by the detonation of $150 \mathrm{~kg}$ of TNT positioned $0.5 \mathrm{~m}$ in front of the column, $2 \mathrm{~m}$ above the ground plane and along the column's centerline. Flexure of concrete and rebar is considered but rebar pull out is not. Figure 4-2 displays the air pressure on a slice plane along the centerline of the deforming column at three time steps. The column is shown with the concrete cutaway in the upper right portion to reveal the deformation of the reinforcing rebar. 


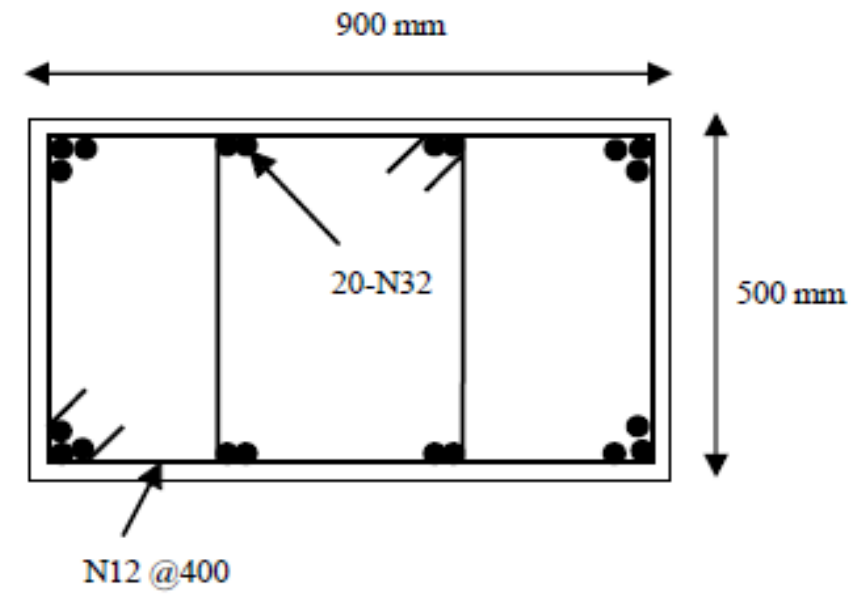

Figure 4-1: Cross-section of column detailing rebar types and locations (400 $\mathrm{mm}$ ligature spacing).

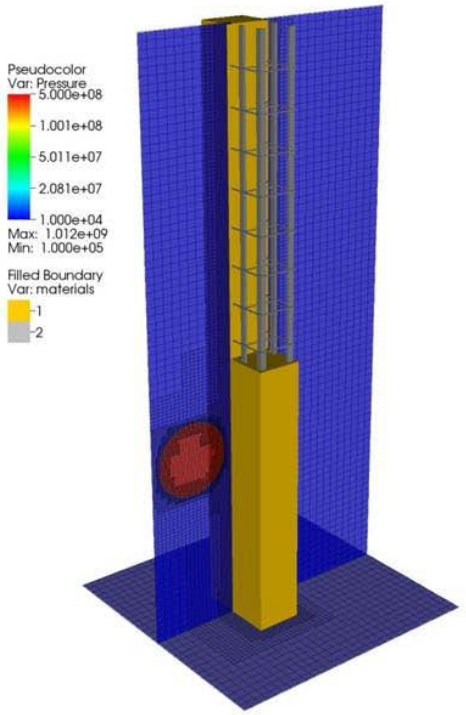

Time $=0.0000 \mathrm{~s}$

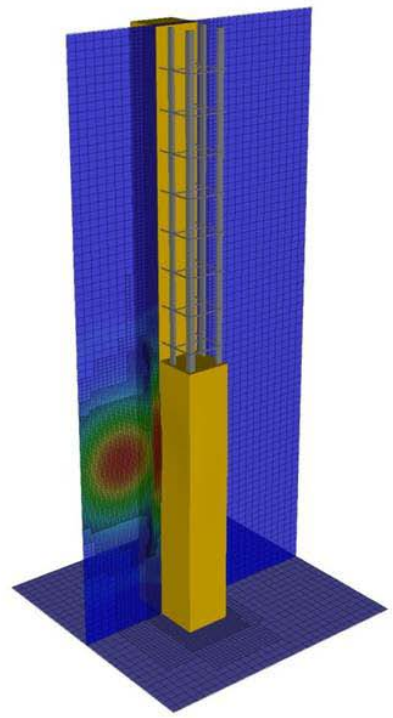

Time $=0.0005 \mathrm{~s}$

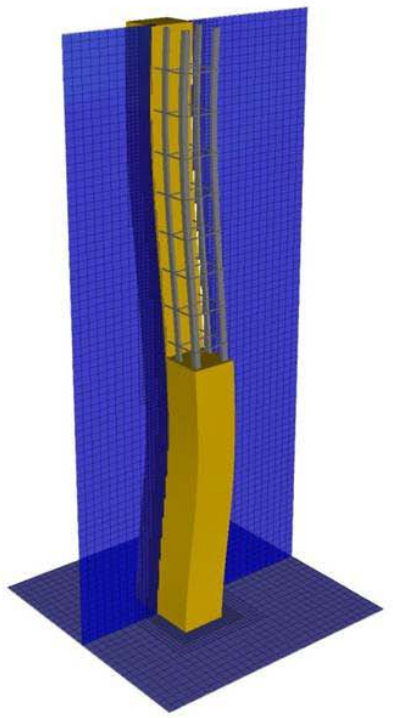

Time $=0.0036 \mathrm{~s}$

Figure 4-2:Air pressure shown on slice planes around deforming column (tan) shown in cutaway to reveal steel rebar (grey) for three time steps. 
$500 \times 900 \mathrm{~mm}$ Reinforced concrete column mesh convergence

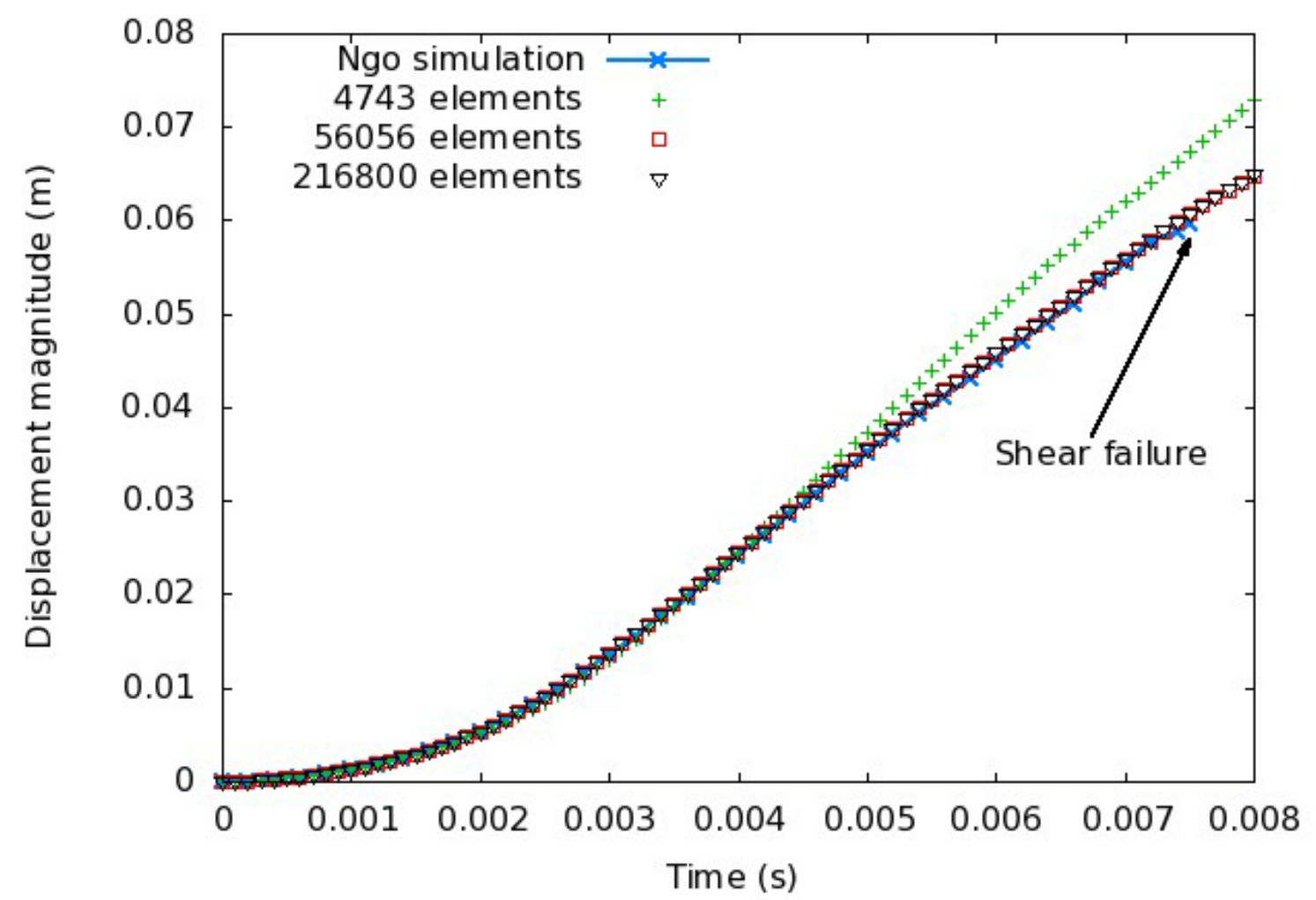

Figure 4-3: Solid mesh convergence and comparison with simulation results by Ngo et al. (2007).

As shown in Figure 4-3 there is less than 5\% of discrepancy between the medium and fine solid mesh refinement levels and results published by Ngo et al. (2007). The simulation in AMROC-DYNA ran for 297 CPU hours on $343.4 \mathrm{GHz}$ Xenon EM64T processors of the Oak Ridge Institutional Cluster (OIC).

\subsubsection{Highway bridge}

This test case follows the setup described by Agrawal and Yi (2009) for blast loading on a typical highway bridge. Figure 4-4 shows the plan and elevation views of the bridge. The internal structure of the bridge deck, the details of the column-deck connection and the rebar reinforcement as described by Agrawal and Yi (2009) were all neglected in this study. The uniform material model for the reinforced structure presented in the disertation was utilized. 

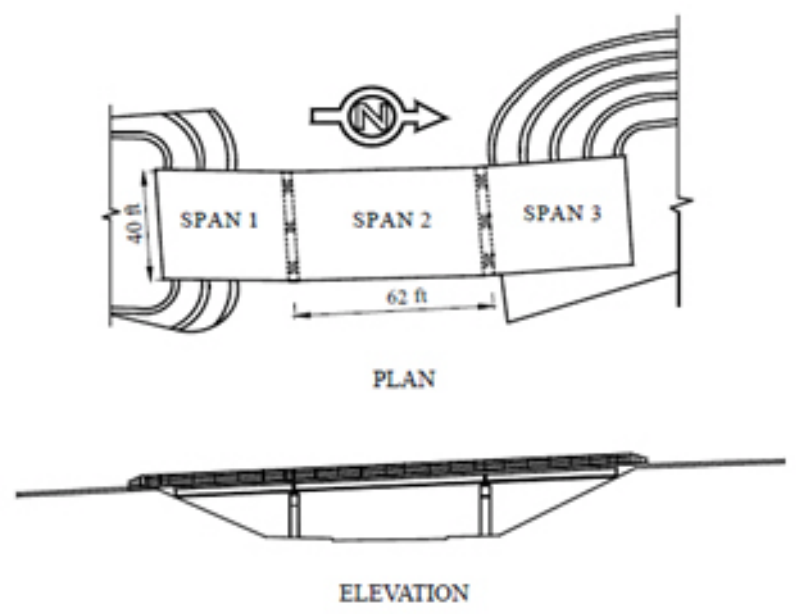

Figure 4-4: Plan and elevation of typical bridge (Agrawal and Yi, 2009).

Figure 4-5 displays the initial time step of the AMROC-DYNA simulation.

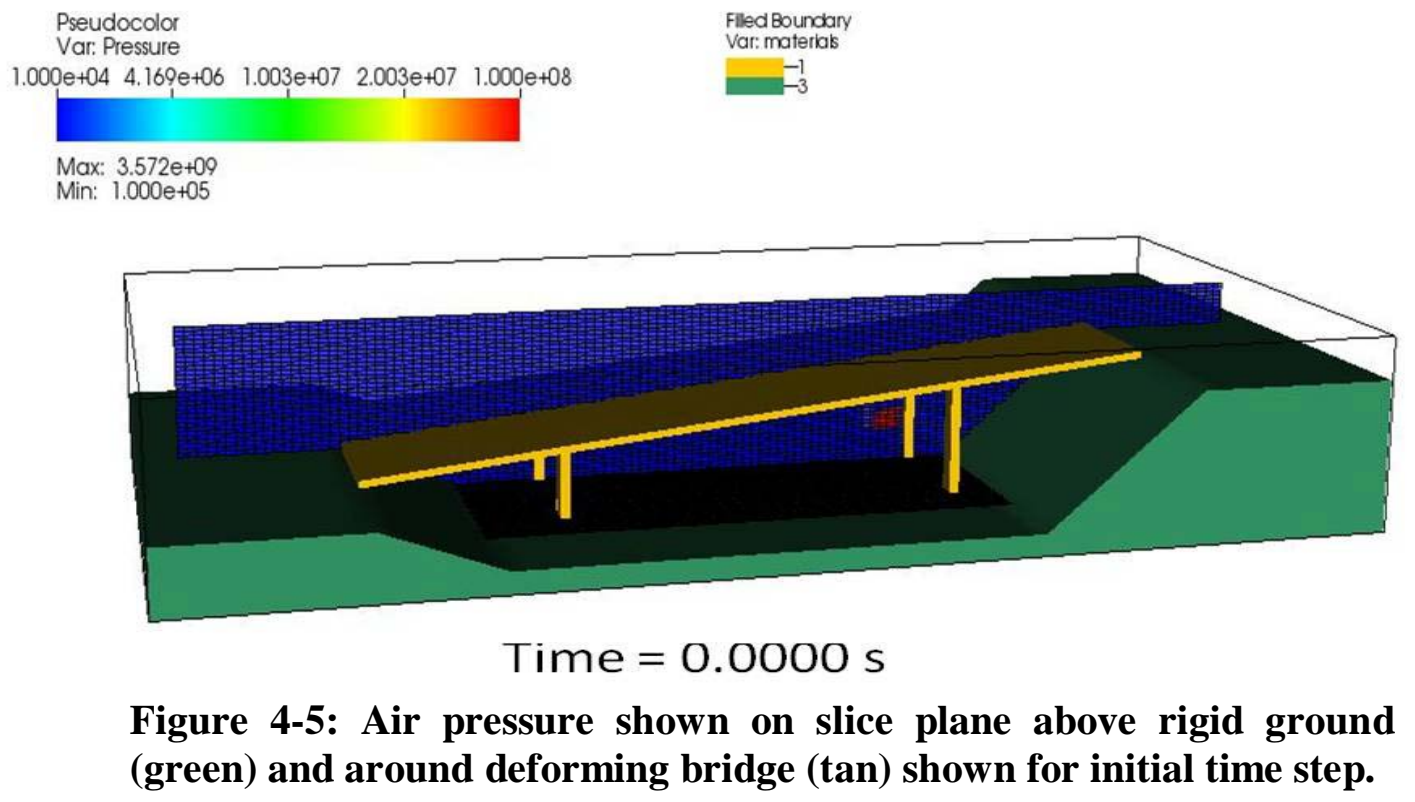

The blast loading is generated by the detonation of $150 \mathrm{~kg}$ of TNT positioned $0.5 \mathrm{~m}$ in front of the central column on the high side of the bridge, $2 \mathrm{~m}$ above the ground plane and along the column's centerline. Figure 4-6 shows the air pressure on a slice plane along the bridge's centerline and on the surfaces of the ground and bridge. Half of the bridge deck is not shown in order to provide a clear view of the propagation of the pressure waves and the adaptive mesh refinement along the slice plane and ground. 


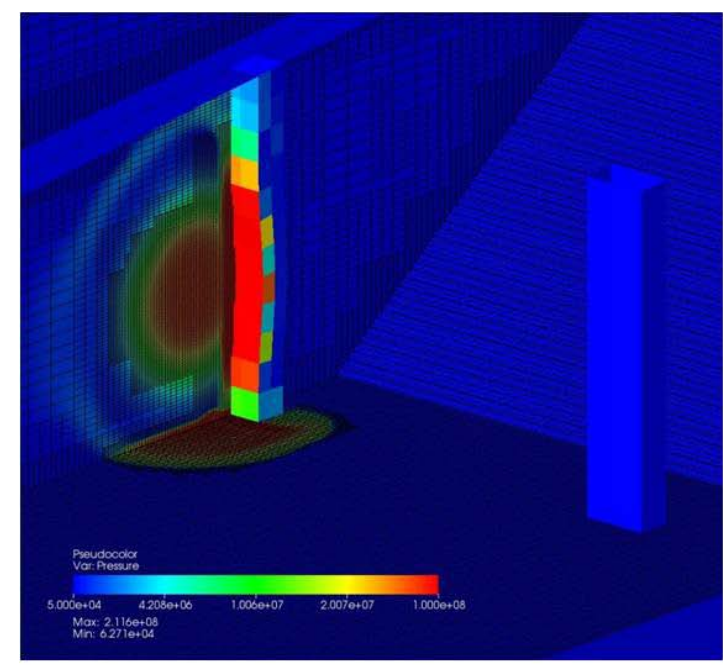

Time $=0.0010 \mathrm{~s}$

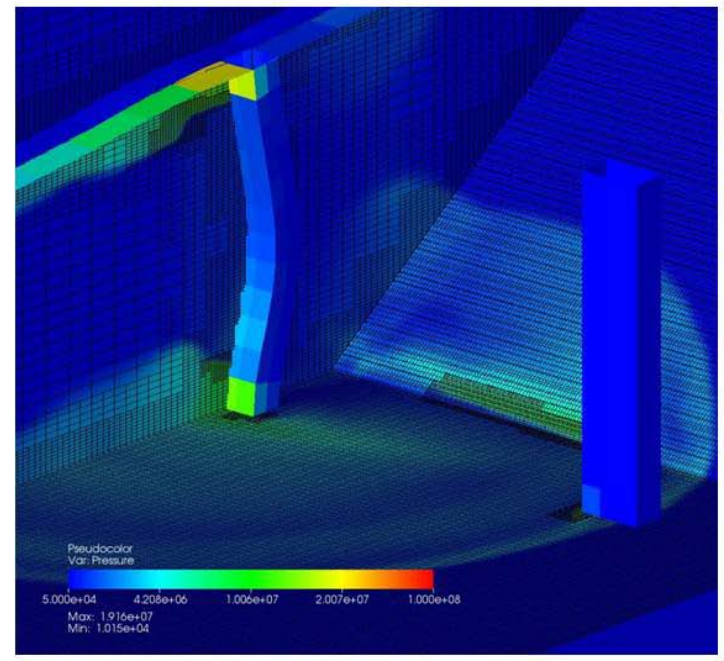

Time $=0.0034 \mathrm{~s}$

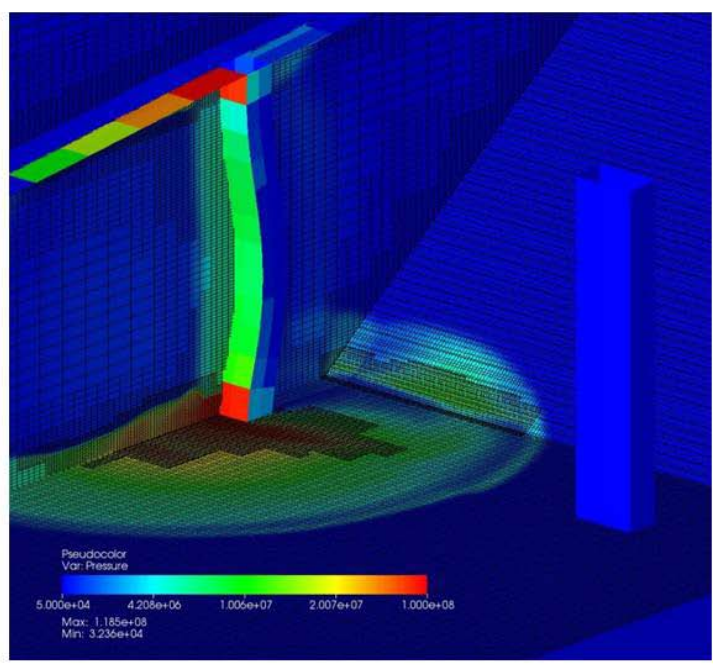

Time $=0.0022 \mathrm{~s}$

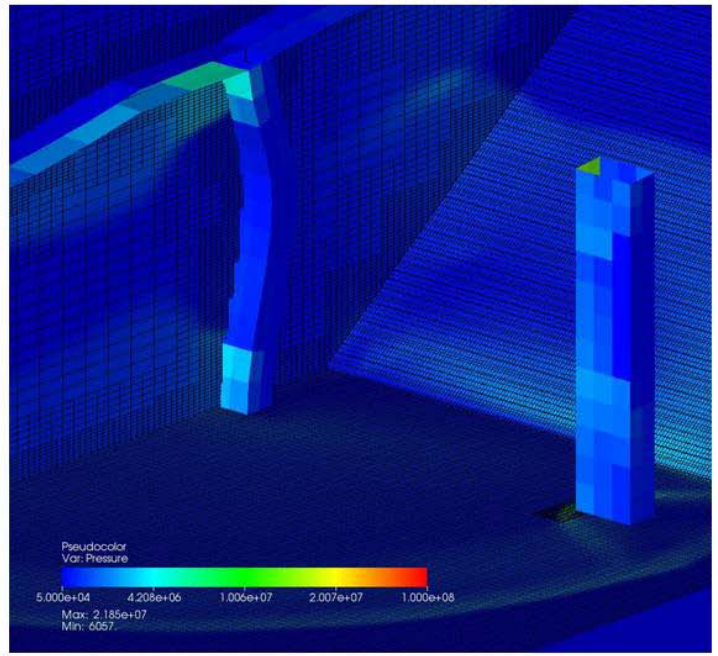

Time $=0.0045 \mathrm{~s}$

Figure 4-6: Air pressure shown on slices of fluid domain and on the surfaces of the bridge and ground (bridge deck removed from view).

There is good agreement between the simplified structural model used in the AMROCDYNA simulation and the results published by Agrawal and Yi (2009). In the work of Agrawal and Yi (2009), structural models of greater complexity were used with a nonadaptive unstructured fluid mesh. The simulation presented here ran for 363 CPU hours on $323.4 \mathrm{GHz}$ Xenon EM64T processors of the Oak Ridge Institutional Cluster (OIC).

Figure 4-7 presents the local deformation and failure in the bridge deck and columns adjacent to the detonation. DYNA's Elastic-Plastic with Failure material model was used to model the simplified bridge. Two failure criteria were utilized: an effective plastic strain based criterion and a hydrostatic tension based criterion. Both criteria are checked at every DYNA time step. If the plastic strain reaches the failure value, $\bar{\varepsilon}_{f}^{p}$, then all deviatoric stresses are set to zero, and the stress state becomes hydrostatic for the 
remainder of the analysis. This hydrostatic stress state may include tensile values which are of smaller magnitude than the failure pressure, $p_{f}$. The pressure criterion is checked even after the element fails due to the strain criterion. If the failure pressure is subsequently exceeded due to hydrostatic tension, the element fails completely and can only support hydrostatic compression (Hallquist and Lin, 2005). Completely failed elements have been removed from Figure 4-7.

The failure criteria used for standard concrete were:

Effective plastic strain at failure, $\bar{\varepsilon}_{f}^{p}=4.010 e-05$

Failure pressure, $p_{f}=-9.100 e+05 \mathrm{~Pa}$

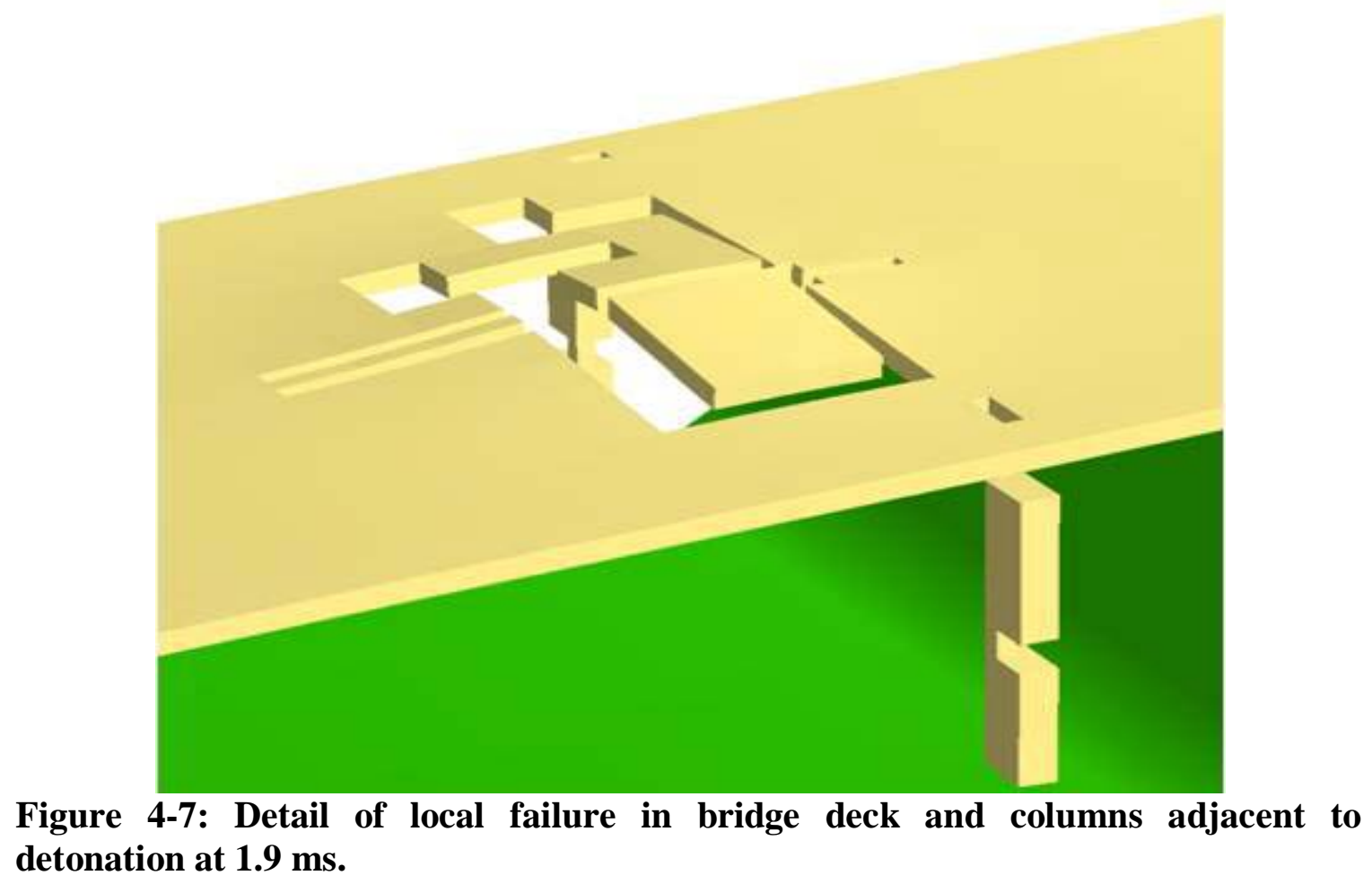

\subsubsection{Multistory building}

This case study is based upon the setup described by Luccioni et al. (2004). The blast event modeled is the detonation of $400 \mathrm{~kg}$ of TNT positioned $1 \mathrm{~m}$ above ground level, $1 \mathrm{~m}$ inside the lobby and $1 \mathrm{~m}$ to the right of the building's centerline. The initial time step of the simulation is shown in Figure 4-8. 


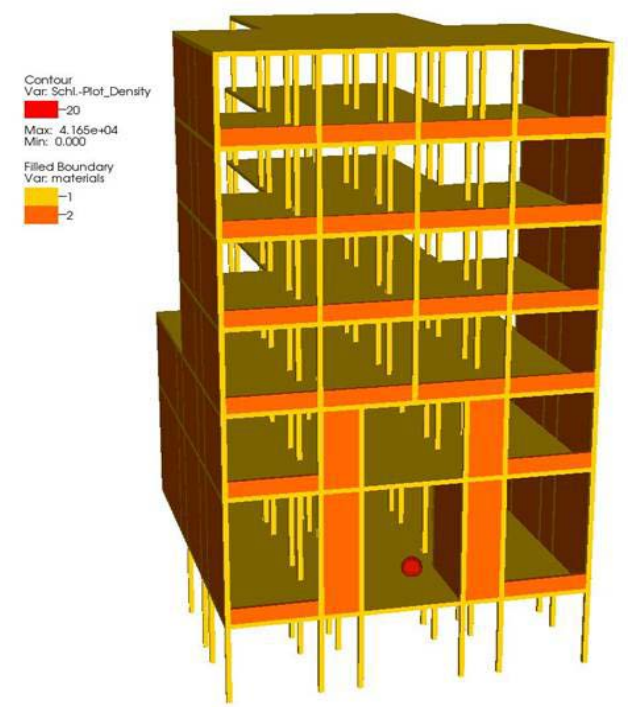

Figure 4-8: Contour of air density gradient (red) shown around deforming building of high strength (yellow) and low strength (orange) concrete for the initial time step.

Two subsequent time steps from the AMROC-DYNA simulation are shown in Figure 4-9.

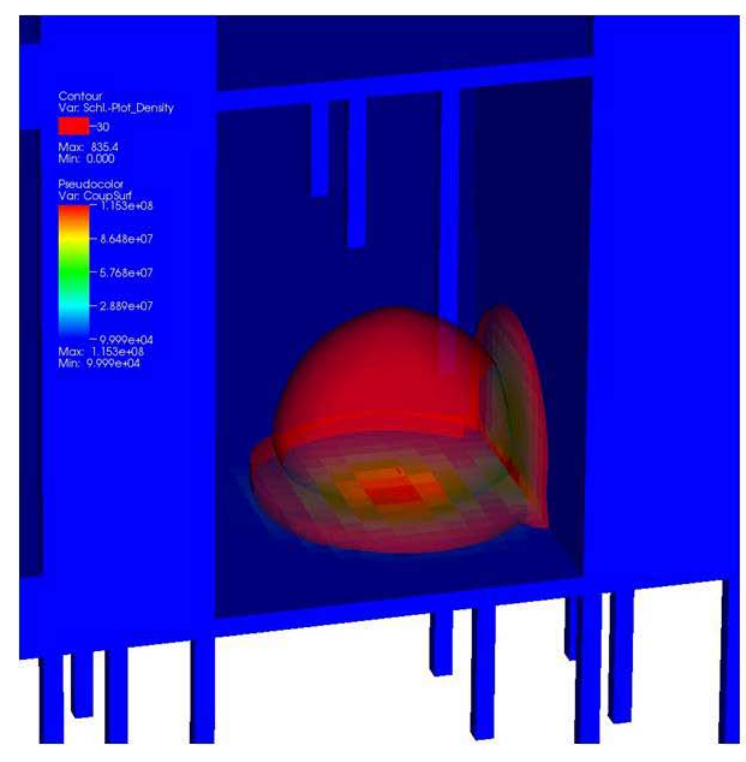

Time $=0.0010 \mathrm{~s}$

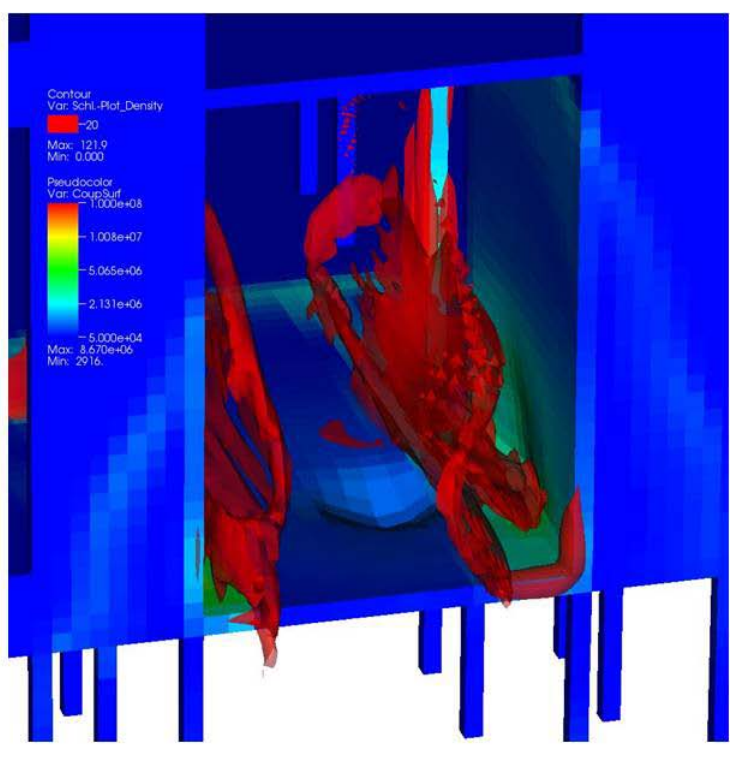

Time $=0.0042 \mathrm{~s}$

Figure 4-9: Contour of air density gradient (red) shown around deforming building colored with surface pressure for two snapshots.

There is good agreement with observations from the aftermath of the explosion and qualitative simulation results published by Luccioni et al. (2004). Figure 4-10 presents the local deformation and failure in the floor, walls and columns adjacent to the detonation. DYNA's Elastic-Plastic with Failure material model was used to model the simplified multistory building. This model is summarized in section 4.1.2 and presented in detail by Hallquist and Lin (2005). 


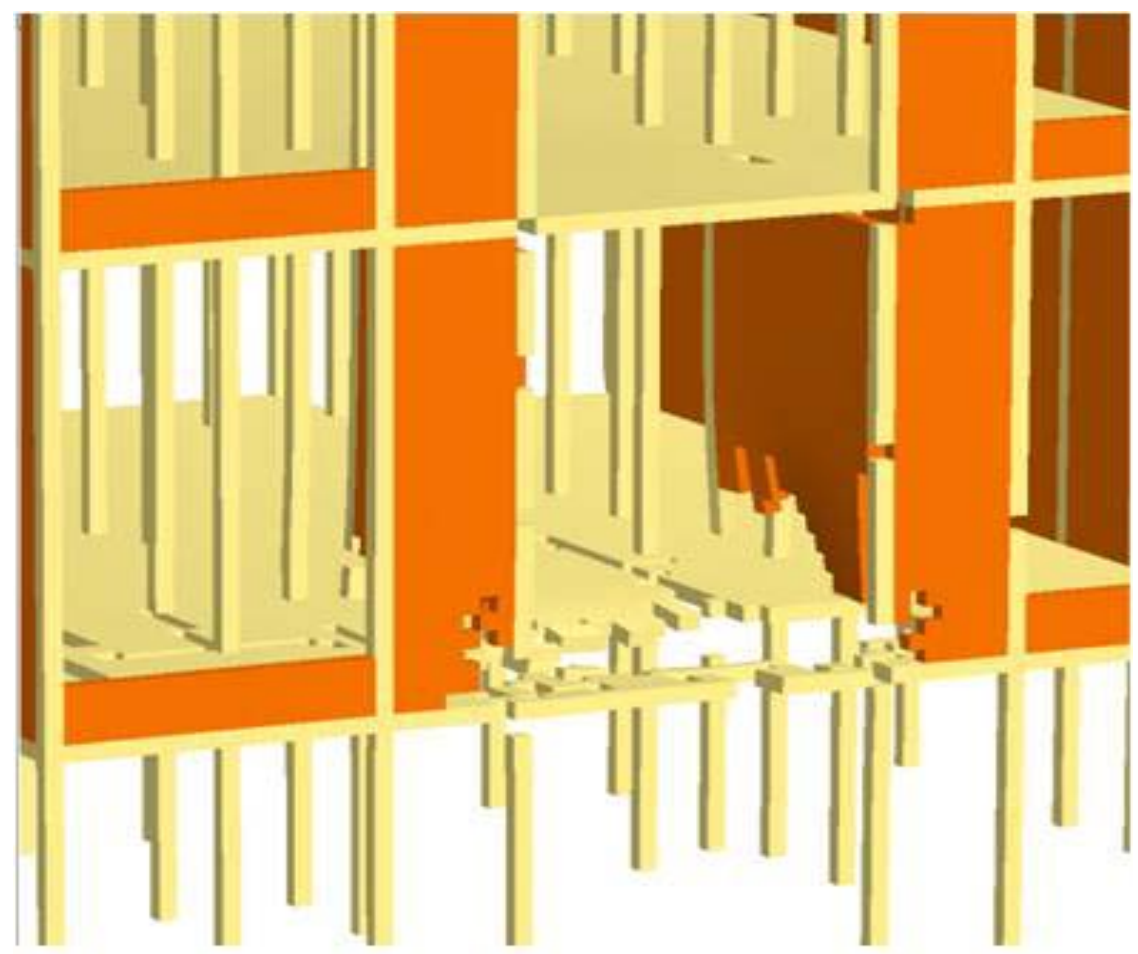

Figure 4-10: Detail of local failure of floors and walls adjacent to detonation at $8 \mathrm{~ms}$.

\subsubsection{Reactor building}

This case study is based upon the General Electric Mark 1 reactors at the Fukushima Daiichi plant. It is intended to demonstrate the ability of AMROC-DYNA to accurately manage FSI calculations in simulations with complex geometry and attachments. For this demonstration a hydrogen explosion initiating above the dryer/separator storage pool has been represented by the detonation of $1000 \mathrm{~kg}$ of TNT.

Figure 4-11 shows the initial time step of the simulation. The forward corner of the reactor building is cut away to reveal the inner structure of the building on lower floors. The forward corner of the superstructure panels are shown translucently. Air pressure is shown on a slice plane along the center line of the reactor and the dryer separator pool. 


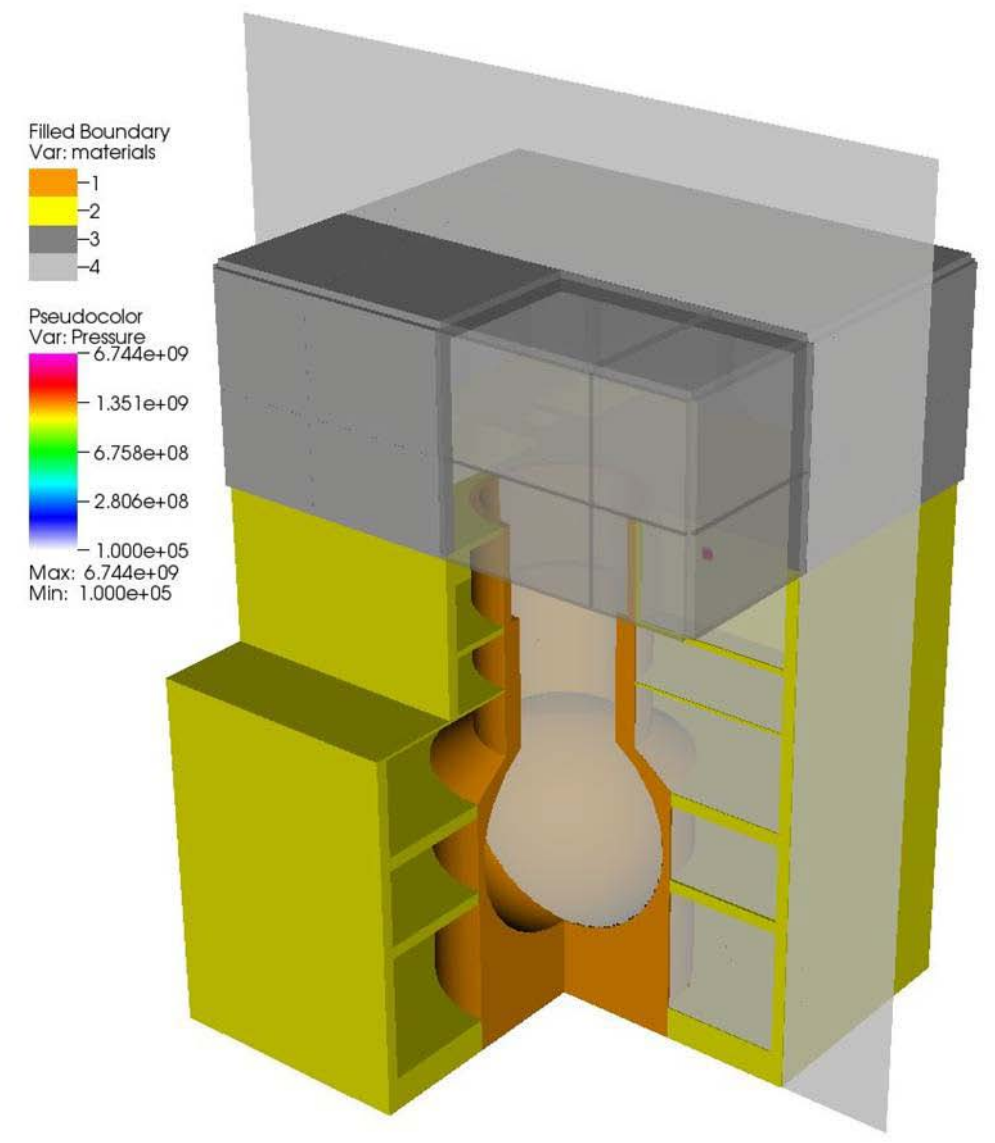

Figure 4-11: Air pressure shown on slice plane around cutout of deforming building of high strength (orange) and low strength (yellow) concrete, girders (dark grey) and paneling (light grey) at initial time.

The attachment between the outer panels and the steel girder superstructure is represented with DYNA's tied with failure sliding interface model. This interface model allows for parts to be joined until a prescribed failure criterion is satisfied, thereafter separation and sliding are allowed. Additionally, DYNA's automatic contact algorithm is utilized to prevent interpenetration of deforming parts. For details of the formulation and implementation of these interface models see (Hallquist and Lin, 2005). 


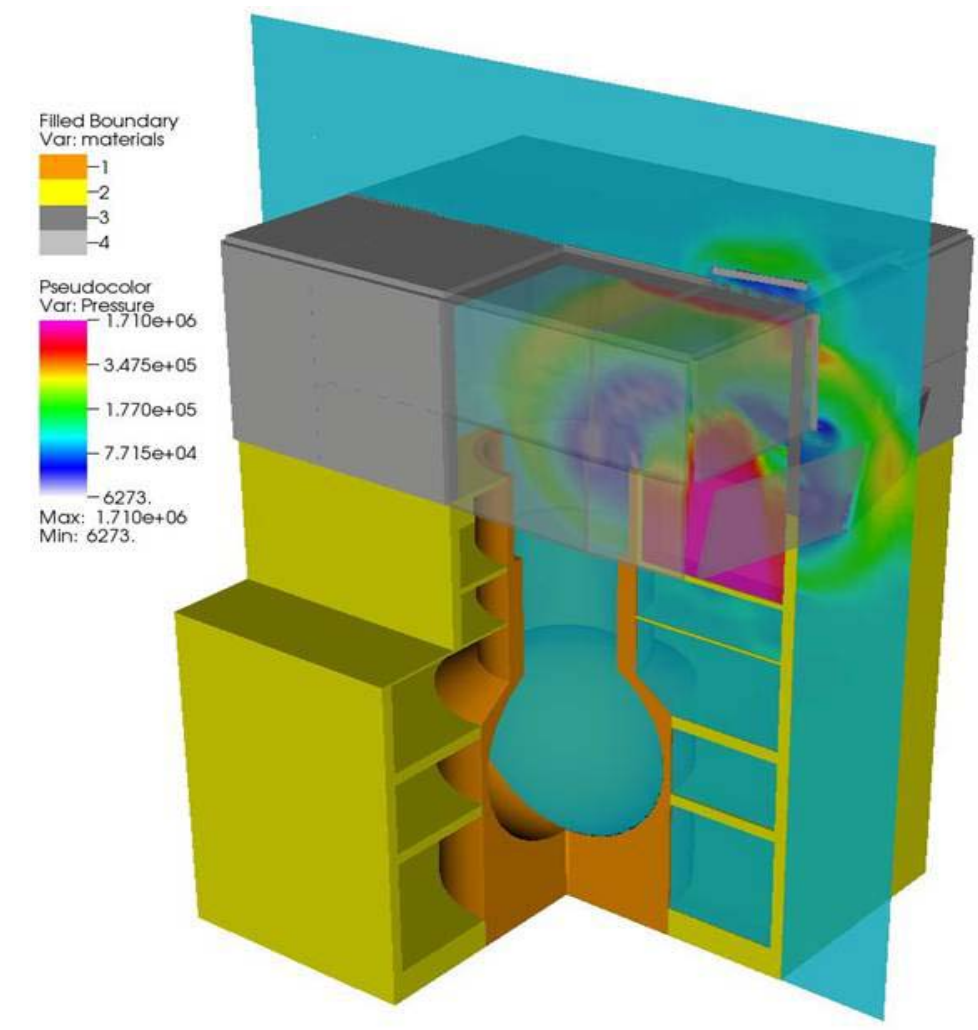

Figure 4-12: Air pressure shown on slice plane around cutout of deforming building of high strength (orange) and low strength (yellow) concrete, girders (dark grey) and paneling (light grey) $1.8 \mathrm{~ms}$ after detonation.

Figure 4-12 and Figure 4-13 show the evolving explosion. The reflection and focusing of shock fronts can be seen in both images along with the motion of the panels as they peel away and separate from the deforming girders. 


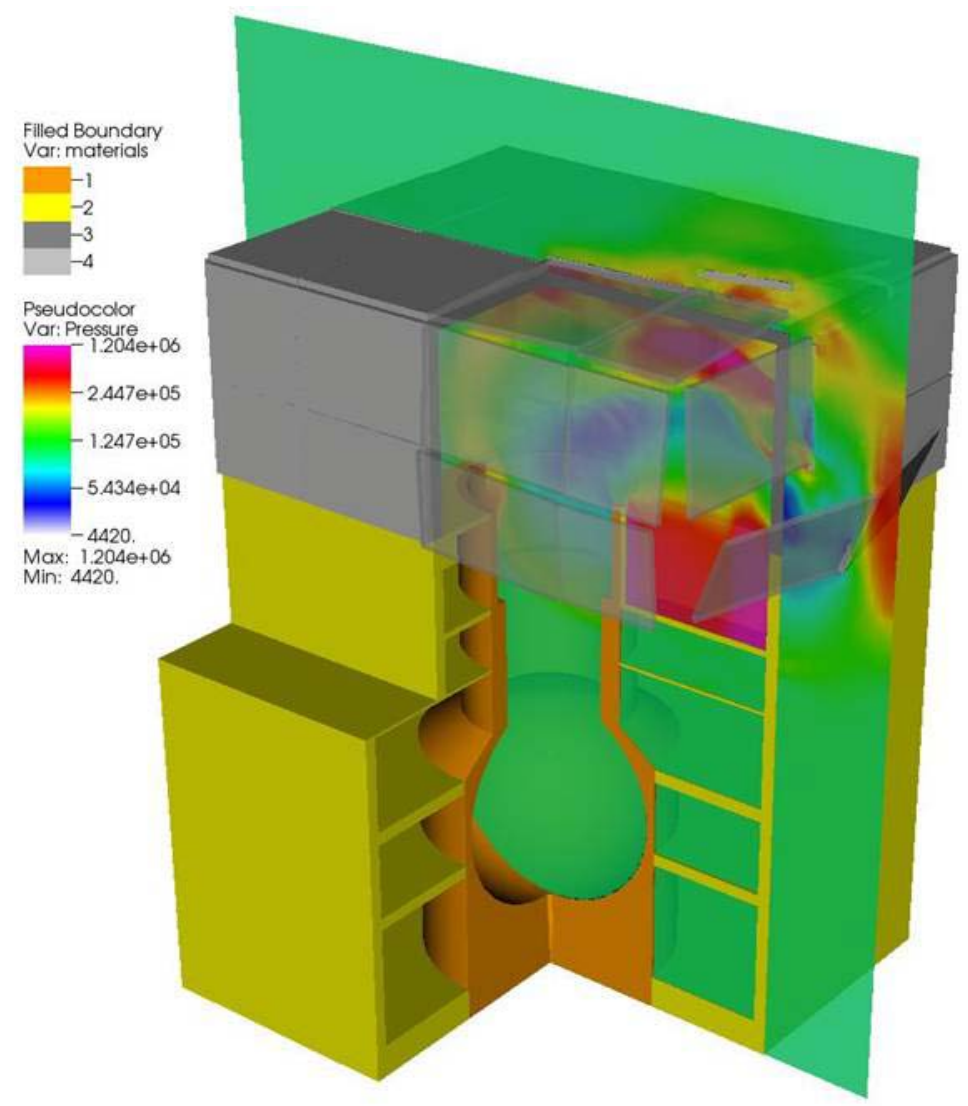

Figure 4-13: Air pressure shown on slice plane around cutout of deforming building of high strength (orange) and low strength (yellow) concrete, girders (dark grey) and paneling (light grey) $2.5 \mathrm{~ms}$ after detonation.

This simulation ran for 3405 CPU hours on $643.4 \mathrm{GHz}$ Xenon EM64T processors of the Oak Ridge Institutional Cluster (OIC).

\section{Conclusions}

AMROC-DYNA is capable of simulating FSI on complex 3D structures as evidenced by the agreement with published simulation results, limited experimental measurements and observations. Material failure and fracture can be modeled and simulated. With its accurate analysis capabilities AMROC-DYNA can be utilized in design and optimization of blast mitigation structures. Further, AMROC-DYNA can be utilized in forensic investigation when necessary.

\section{Future work}

Development of the coupling surface routine to enable large deformations and fragmentation following failure will be continued. Further development of hydrogen detonation models is planned during the next year. Incorporation of case appropriate fluid and material properties into required models along with a material model parameter study is also planned during the next year. 


\section{References}

Agrawal, A. K., \& Yi, Z. (2009). Blast Load Effects on Highway Bridges. New York: University Transportation Research Center City Colege of New York.

Arienti, M., Hung, P., Morano, E., \& Shepherd, J. E. A level set approach to EulerianLagrangian copuling. J. Comput. Phys., 185:213-251, 2003.

Berger, M. \& Colella, P. Local adaptive mesh refinement for shock hydrodynamics. J. Comput. Phys., 82:64-84, 1988.

Cirak, F., Deiterding, R., \& Mauch, S. P. Large-scale fluid-structure interaction simulation of viscoplastic and fracturing thin shells subjected to shocks and detonations. Computers \& Structures, 85(11-14):1049-1065, 2007.

Deiterding, R. Parallel adaptive simulation of multi-dimensional detonation structures. $\mathrm{PhD}$ thesis, Brandenburgische Technische Universität Cottbus, Sep 2003.

Deiterding, R. Construction and application of an AMR algorithm for distributed memory computers. In T. Plewa, T. Linde, and V. G. Weirs, editors, Adaptive Mesh Refinement - Theory and Applications, volume 41 of Lecture Notes in Computational Science and Engineering, pages 361-372. Springer, 2005.

Deiterding, R. A parallel adaptive method for simulating shock-induced combustion with detailed chemical kinetics in complex domains. Computers \& Structures, 87:769783, 2009.

Cirak, F., Deiterding, R., \& Mauch, S. P. Efficient fluid-structure interaction simulation of viscoplastic and fracturing thin-shells subjected to underwater shock loading. In S. Hartmann, A. Meister, M. Schäfer, and S. Turek, editors, Int. Workshop on FluidStructure Interaction. Theory, Numerics and Applications, Herrsching am Ammersee 2008, pages 65-80. kassel university press GmbH, 2009.

Deiterding, R., Radovitzki, R., Mauch, S. , Cirak, F., Hill, D. J., Pantano, C., Cummings, J. C., \& Meiron, D. I. Virtual Test Facility: A virtual shock physics facility for simulating the dynamic response of materials. Available at http://www.cacr.caltech.edu/asc.

Deiterding, R. Anais do CNMAC, Vol. 3, Proc. CNMAC 2010 - 33rd Brazilian National Congress for Applied and Computational Mathematics, pages 1048-1057, 2010.

Deiterding, R., Cirak, F. \& Mauch, S. Int. Workshop Fluid-Structure Interaction. Theory, Numerics and Applications, Herrsching am Ammersee, pages 65-80, kassel university press GmbH, Kassel, 2009.

Deiterding, R., Radovitzki, R., Mauch, S. , Noels, L., Cummings, J. C., \& Meiron, D. I. A virtual test facility for the efficient simulation of solid materials under high energy shock-wave loading. Engineering with Computers, 22(3-4):325-347, 2006.

Fedkiw, R. P. Coupling an Eulerian fluid calculation to a Lagrangian solid calculation with the ghost fluid method. J. Comput. Phys., 175:200-224, 2002.

Fedkiw, R. P. , Aslam, T., Merriman, B., \& Osher, S. A non-oscillatory Eulerian approach to interfaces in multimaterial flows (the ghost fluid method). J. Comput. Phys., 152:457-492, 1999.

Hallquist, J. \& Lin, J. I. A nonlinear explicit three-dimensional finite element code for solid and structural mechanics. Technical Report UCRL-MA-107254, Lawrence Livermore National Laboratory, 2005. Source code (U.S. export controlled) available for licensing fee from http://www.osti.gov/estsc. 
LeVeque, R. J. Finite volume methods for hyperbolic problems. Cambridge University Press, Cambridge, New York, 2002.

Lin, J. I. DYNA3D: A Nonlinear, Explicit, Three-Dimensional Finite Element Code for Solid and Structural Mechanics - User Manual, Lawrence Livermore National Laboratory, 2005.

Longinow A, and Mniszewski KR., Protecting buildings against vehicle bomb attacks. Practice Periodical on Structural Design and Construction, ASCE, New York, pp. 51-54, 1996.

Löhner, R., Baum, J., Charman, C., \& Pelessone, D. Fluid-structure interaction simulations using parallel computers. volume 2565 of Lecture Notes in Computer Science, pages 3-23. Springer, 2003.

Löhner, R., Cerbal, J., Yang, C., Baum, J. D., Mestreau, E., Charman, C., et al. (2004). Large-Scale Fluid-Structure Interaction Simulations. Computing in Science \& Engineering , 6 (3).

Luccioni, B. M., Ambrosini, R. D., \& Danesi, R. F. (2004) Analysis of building collapse under blast loads. Engineering Structures 26, pages 63-71

Ngo, T., Mendis, P., Gupta, A., \& Ramsay, J. (2007). Blast Loading and Blast Effects on Structures - An Overview. Electronic Journal of Structual Engineering .

Mauch, S. P. Efficient Algorithms for Solving Static Hamilton-Jacobi Equations. PhD thesis, California Institute of Technology, 2003.

Mittal, R. \& Iaccarino, G. Immersed boundary methods. Annu. Rev. Fluid Mech., 37:239-261, 2005.

Parashar, M. \& Browne, J. C. On partitioning dynamic adaptive grid hierarchies. In Proc. of the 29th Annual Hawaii Int. Conf. on System Sciences, Jan 1996.

TM 5-1300, The Design of Structures to Resist the Effects of Accidental Explosions, Technical Manual, US Department of the Army, Navy, and Air Force, Washington DC, 1990.

Toro, E. F. Riemann solvers and numerical methods for fluid dynamics. Springer-Verlag, Berlin, Heidelberg, 2nd edition, 1999.

Toro, E. F., Spruce, M., \& Speares, W. Restoration of the contact surface in the HLLRiemann solver. Shock Waves, 4:25-34, 1994. 